\title{
Acknowledgement to Reviewers of Life in 2018
}

\section{Life Editorial Office}

MDPI, St. Alban-Anlage 66, 4052 Basel, Switzerland

Published: 9 January 2019

\begin{abstract}
Rigorous peer-review is the corner-stone of high-quality academic publishing. The editorial team greatly appreciates the reviewers who contributed their knowledge and expertise to the journal's editorial process over the past 12 months. In 2018, a total of 65 papers were published in the journal, with a median time to first decision of 16 days and a median time to publication of 43 days. The editors would like to express their sincere gratitude to the following reviewers for their cooperation and dedication in 2018:
\end{abstract}

Altwegg, Kathrin

Amor, Daniel R.

Ares, Saúl

Arnold, Roland

Bada, Jeff

Bains, William

Bej, Asim

Belinky, Frida

Benison, Kathleen

Benner, Christopher

Bernhardt, Harold S.

Bevilacqua, Antonio

Billette, Christophe

Blanco, Celia

Boto, Luis

Botta, Lorenzo

Bouaïcha, Noureddine

Brack, André

Buckel, Wolfgang

Burton, Aaron S.

Cafferty, Brian

Carro, Lorena

Cavalazzi, Barbara

Chatzitheodoridis, Elias

Chen, Irene

Cintas, Pedro

Cleaves, Henderson James

Conde-Pueyo, Nuria

Corbí, Hugo

Cordero, Radames

Cowan, Don

Damer, Bruce

Danelon, Christophe

Daniel, Isabelle

De La Varga, Herminia
De Los Santos, Emmanuel Lorenzo

Deamer, David

Degnan, Patrick

Delaye, Luis

Delbarre-Ladrat, Christine

DeVeaux, Linda C.

Dhakal, Dipesh

D'Hendecourt, Louis

Di Giulio, Massimo

Di Mauro, Ernesto

Dixon, Nicholas

Doyle, Vinson

Egel, Richard

Ehira, Shigeki

Erastova, Valentina

Etxebeste, Oier

Facciotti, Marc T.

Flores García, Enrique

Forchhammer, Karl

Fornaro, Teresa

Foucher, Frédéric

Fujihara, Akimasa

Fujii, Hodaka

Fujita, Yuichi

Furukawa, Yoshihiro

Gómez-Baena, Guadalupe

Gonçalves, Luís Gafeira

Goñi-Moreno, Angel

Gonzales, Rafael Navarro

Gooley, Paul

Gottlieb, Alexandra

Grishkan, Isabella

Gueidan, Cécile

Guespin, Janine

Gutarowska, Beata 
Higgs, Paul

Hsu, Fang Rong

Huang, Hao

Jheeta, Sohan

Jia, Tony

Jimenez, M. Angeles

Judit, Šponer

Kaddour, Hussein

Kalsi, Megha

Karunakaran, Suneesh

Kerou, Melina

Kiga, Daisuke

King, Helen

Kirkendall, Lawrence

Kirschner, Roland

Kitadai, Norio

Kohno, Nobuaki

Kolb, Vera

Kooij, Pepijn W.

Kun, Adam

Kunnev, Dimiter

Kwon, Yongchan

Lebrun, Vincent

Lehman, Niles

Leuko, Stefan

Liu, Allen

Lo Giudice, Angelina

Lücking, Robert

Lueth, Virgil W.

Maldener, Iris

Mancinelli, Rocco L

Mansy, Sheref

Mao, Meng

Marnocha, Cassandra

Massa, Gioia

Maurel, Marie-Christine

Mayer, Christian

Mayhew, Lisa E.

Michaelian, Karo

Mirete, Salvador

Molina-Heredia, Fernando P.

Montalvo-Rodríguez, Rafael

Montañez Martinez, Raul

Moore, Allen J.

Moran, Joseph

Moras, Dino

Morton, Cynthia M.

Mullineaux, Conrad W.

$\mathrm{Na}$, Dokyun

Naraoka, Hiroshi

Nelsen, Matthew P.

Newman, Jeffrey
Nikoh, Naruo

Norris, Vic

Ntougias, Spyridon

Nuevo, Michel

Olabarriaga, Silvia D.

Oliveira, Paulo

Oren, Aharon

Orlando, Thomas M.

Paleos, Constantinos M.

Pangallo, Domenico

Pascal, Robert

Pasek, Matthew

Pedreira-Segade, Ulysse

Peltzer, Alexander

Perez-Carrasco, Ruben

Piel, William H

Pinheiro, Vitor

Pinter, Tyler

Potvin-Trottier, Laurent

Powner, Matthew

Pucciarelli, Sandra

Ragusa, Maria Antonietta

Rahm, Martin

Ranjan, Sukrit

Rimmer, Paul

Robb, Craig

Rogers, Jeffrey

Ross, David S.

Rudolph, Christian

Ruiz-Bermejo, Marta

Ruiz-Mirazo, Kepa

Russell, Michael J.

Sahai, Nita

Saitta, Antonino Marco

Sakamoto, Kensaku

Schirmack, Janosch

Schrenk, Matthew

Shain, Daniel H.

Shawn, McGlynn

Shimizu, Kazuyuki

Singh, Nitin K

Sonnino, Giorgio

Šponer, Jiří

Stano, Pasquale

Strassmann, Joan

Strazewski, Peter

Succurro, Antonella

Sun, Fengjie

Surman, Andrew J.

Takashima, Masako

Takezaki, Naoko

Tan, Cheemeng 
Tessera, Marc

Thiel, Teresa

Treu, Roland

Trindle, Carl

Tyystjärvi, Taina

Vallée, Yannick

Van Doninck, Karine

Vaverková, Magda

Vavitsas, Konstantinos
Videau, Patrick

Walde, Peter

Wieczorek, Rafal M.

Wolk, Coleman Peter

Zabel, Paul

Zaritsky, Arieh

Zentner, Gabriel

Zhang, Cheng-Cai

Zuo, Ran

(C) 2019 by the author. Licensee MDPI, Basel, Switzerland. This article is an open access article distributed under the terms and conditions of the Creative Commons Attribution (CC BY) license (http://creativecommons.org/licenses/by/4.0/). 